\title{
Quaderni
}

QUADERNI Communication, technologies, pouvoir

70 | Automne 2009

Think tanks, experts et pouvoirs

\section{Avant-propos : think tanks, experts et pouvoirs}

\section{Geoffrey Geuens}

\section{OpenEdition}

Journals

Édition électronique

URL : http://journals.openedition.org/quaderni/502

DOI : 10.4000/quaderni.502

ISSN : 2105-2956

\section{Éditeur}

Les éditions de la Maison des sciences de l'Homme

\section{Édition imprimée}

Date de publication : 5 octobre 2009

Pagination : 5-9

\section{Référence électronique}

Geoffrey Geuens, "Avant-propos : think tanks, experts et pouvoirs », Quaderni [En ligne], 70 | Automne 2009, mis en ligne le 05 octobre 2011, consulté le 22 septembre 2020. URL : http://

journals.openedition.org/quaderni/502 ; DOI : https://doi.org/10.4000/quaderni.502 


\section{$D$ ossier}

\section{avant-propos : think tanks,} experts et pouvoirs

Des dossiers de presse à la littérature professionnelle, en passant par les sites Internet, essais semisavants et autres colloques, il ne manque pas de signes témoignant aujourd'hui de la consécration symbolique du monde des think tanks dans le discours social. Ces institutions, le plus souvent privées, rencontrent d'une part, l'intérêt toujours croissant d'un monde académique anglo-saxon largement au fait de ces réalités - aux États-Unis, les travaux précurseurs dans le domaine datent des premières années de la guerre froide, époque où apparaît d'ailleurs le terme de « think tank » lui-même ${ }^{1}$ - mais ont également réussi à éveiller la curiosité des journalistes, des décideurs et des chercheurs francophones en sciences humaines dont l'intérêt se limitait, jusqu'il y a peu, à la sociologie des intellectuels, l'histoire des écoles

Chargé de cours, département des Arts et Sciences de la Communication, Université de Liège et doctrines ou encore l'analyse de l'expertise publique. Si les think tanks font ainsi l'objet d'une hypertrophie médiatique, les travaux et controverses qu'ils suscitent n'en témoignent pas moins d'un sentiment diffus de confusion et de fermeture du débat auquel devrait répondre ce nouveau dossier de la revue Quaderni.

Sommes-nous bien sûrs, tout d'abord, de savoir de quoi il s'agit réellement? Traduit par les termes « réservoir à penser », « boîte à idées » ou « cercle de réflexion », le think tank désigne communément la réunion d'un ensemble de personnes et/ou de ressources intellectuelles engagées dans la recherche, la production et la diffusion d'analyses en politiques publiques à destination des autorités et, en certains cas même, des entreprises et des organisations non gouvernementales. Cette définition, pour évidente qu'elle apparaisse, demande néanmoins à être interrogée à la lumière des objectifs et pratiques dont témoignent géné- 
ralement les think tanks. Si le phénomène fascine et interpelle, c'est peut-être au plus visible par la variété des institutions que recouvre le vocable en question, qu'il s'agisse d'organismes publics de recherche (Conseil d'analyse économique, Centre d'études prospectives et d'informations internationales), de fondations politiques (Fondation pour l'innovation politique, Fondation Jean Jaurès, Fondation Robert Schuman), de groupes de réflexion patronaux (Institut de l'entreprise) ou d'autres organisations ouvertement partisanes (Association pour la liberté économique et le progrès social). Quoi de commun en effet sur le plan de l'autonomie intellectuelle entre un centre universitaire d'études comme l'Observatoire français des conjonctures économiques - dépendant de Sciences Po - et à l'autre extrême un advocacy group tel que le Project for a New American Century, chargé de justifier auprès de l'opinion les plans de «domination globale» imaginés par une poignée de néo-conservateurs? Il convient en outre de noter que cette confusion des genres est entretenue par le fait que certains think tanks sont eux-mêmes généralement traversés par de multiples logiques institutionnelles, leurs structures dirigeantes notamment relevant parfois simultanément du cénacle académique, du club politique et du lobby industriel.

L'abondance des publications consacrées aux think tanks est ensuite trompeuse à bien des égards. D'un côté, à y regarder de plus près, l'on s'apercevrait sans grande difficulté que l'ensemble de ces travaux se laissent largement distribuer sous trois grandes catégories génériques : celle des annuaires prétendant à la pure factualité descriptive, qu'ils soient le fait d'historiens ou de journalistes d'investigation; celle ensuite d'ouvrages de vulgarisation portés par une ferveur militante - célébrant sur le mode managérial la créativité, l'innovation et le pragmatisme d'une nouvelle intelligentsia - ou dénonçant au contraire la tyrannie des experts, l'imposition d'une pensée unique et la censure de l'espace public par des pouvoirs privés illégitimes ; celle enfin d'écrits à vocation philosophique, sociologique ou politologique rendant compte des relations d'interdépendance réciproque entre think tanks, pouvoir(s) et société. Ces auteurs et ces textes valent moins par conséquent comme ressources théoriques que comme objets ou discours à interroger, dont un autre principe de rareté sous couvert d'abondance apparaît clairement, à savoir que, à quelle que catégorie qu'ils appartiennent, l'ensemble formé par ces discours se confond largement avec l'orchestration d'un petit nombre de lieux communs en décrochage avec les grands référentiels passés. La problématique des think tanks est ainsi organiquement liée à l'idée de " société civile», concept fourre-tout s'inscrivant actuellement dans un imaginaire tout à fait singulier : celui de la société de la gouvernance, instituée en paradigme scientifique, et renvoyant dans son acception commune à la description d'un monde de plus en plus « complexe », caractérisé par l'avènement de nouveaux acteurs intégrés au processus de décision (think tanks, ONG...) et par la substitution de l'expert au politique.

Signe de modernité pour les uns, d'impérialisme culturel pour les autres, l'apparition en France du terme think tank - qui tient de l'effet de mode autant que d'imposition - est d'ailleurs ellemême inséparable de l'émergence du concept de « politiques publiques » importé de la culture managériale anglo-saxonne (« Public Policy »). 
Tendant systématiquement à surestimer le pouvoir de l'industrie privée de la consultance, les analyses en termes de «stateless global governance » pèchent en outre par leur propension à ne voir dans les think tanks que des concurrents, loyaux ou déloyaux, des pouvoirs publics. Et cela alors même que l'État français - pour ne prendre que ce seul exemple - a favorisé de facto l'émergence des boîtes à idées spécialisées dans le domaine de la politique étrangère ${ }^{2}$. Quoiqu'il en soit, adversaires et partisans de la révolution des think tanks participent donc, à leur corps défendant, d'une même structure bipolaire - État vs société civile - indissolublement liés, dirait Lucien Sfez, «par la querelle et le dispositif unique qui les gouverne $»^{3}$.

La question des think tanks demeure enfin aujourd'hui aussi peu questionnée dans sa généalogie concrète, et notamment sa genèse sociale, que dans sa substance logique et son poids de réalité. Ce n'est pourtant pas un hasard si le regain d'intérêt pour l'étude de ces institutions coïncide, au début des années 1990, avec la redécouverte des vertus associées à la société civile - notamment à l'Est, mais pas seulement - et si un grand nombre d'influentes boîtes à idées sont nées dans des contextes de transformation majeure de nos sociétés. Des débats sur la paix (Royal Institute for International Affairs, 1920) aux controverses européennes (Centre for European Policy Studies, 1983) en passant par la « révolution » libérale (Institut de l'Entreprise, 1975), chaque crise économique, politique ou culturelle voit l'émergence de nouveaux groupes de réflexion - plus ou moins institutionnalisés - et d'experts « pragmatiques » chargés d'apporter des solutions « innovantes » aux problématiques du moment.
Cette représentation techniciste, apolitique et par là même neutralisante de la consultance ne résiste pourtant pas longtemps à l'analyse empirique. C'est que l'étude ciblée des principales boîtes à idées nord-américaines et européennes témoigne d'un processus d'hétéronomisation croissante du champ intellectuel.

Le présent dossier va donc s'attacher à ouvrir le débat sur les think tanks en évitant autant le parti pris politique que les polémiques stériles ou les effets de mode fréquents en la matière. Les temps semblent en effet mûrs pour réfléchir à cette problématique, et cela dans la mesure où ces institutions font aujourd'hui encore essentiellement l'objet d'une littérature non académique, si l'on excepte les quelques auteurs de référence dans le domaine - songeons à Diane Stone, Mark Garnett, Donald Abelson, James McGann ou encore Andrew Denham ${ }^{4}$ - mais dont les travaux se limitent le plus souvent à l'univers anglo-saxon. Le présent dossier s'articulera autour de trois axes de réflexion.

Le premier volet prendra la forme d'une conceptualisation de la problématique en question. Il s'agira notamment, dans cette perspective, d'interroger les enjeux politiques des débats portant sur les éléments définitoires du concept de « think tank» : Quelles institutions réclament ou se réclament de ce « label»? Et quels sont les profits symboliques associés à cette dénomination spécifique ? C'est que les discours d'autocélébration légitimante ont à faire avec des questions de visibilité et d'identité, et cela dans les affirmations (indépendance, transparence) comme dans les dénégations (groupe politique, lobby) des dirigeants des boîtes à idées. Les pa- 
roles d'experts labellisés think tankers participent en outre - on le verra - à un processus circulaire de légitimation réciproque entre décideurs, médias et consultants (Lucile Desmoulins).

Le deuxième volet de ce dossier s'efforcera de mieux saisir le rôle de l'expertise privée dans la dissémination des idées, en prenant bien évidemment en compte les contextes institutionnels nationaux au sein desquels les think tanks ont à opérer. Une organisation telle que la Rand Corporation - née dans le giron de l'industrie de la défense - a ainsi participé activement au façonnement de la futurologie et à l'imposition du paradigme de la " société de l'information », le tout sur fond de «fin des idéologies » et de révolution technique permanente (David Forest). Nombreux d'ailleurs sont les facteurs institutionnels expliquant cette influence considérable des think tanks dans le champ américain des politiques publiques. Au plan national, deux traits essentiels du système politique des États-Unis doivent être pointés : d'une part, l'éclatement de l'administration fédérale et la perméabilité à la société civile, d'autre part, le manque de cohésion idéologique des partis politiques engendrant une multiplication des demandes d'expertise (Daniel Béland). Une comparaison systématique du Council on Foreign Relations et de l'Institut français des Relations internationales atteste d'ailleurs bien de l'importance de l'environnement institutionnel dans la légitimation ou la non- légitimation des acteurs diplomatiques privés (François Vergniolle de Chantal).

Un dernier volet s'attachera enfin à repenser la position des think tanks dans l'espace du pouvoir, et cela en évitant l'écueil de l'opposition stérile
« État vs société civile », qu'il s'agisse pour les uns d'invoquer l'autonomie des boîtes à idées à l'égard du politique, ou pour d'autres de dénoncer - plus communément - l'instrumentalisation des groupes de réflexion par le gouvernement. Aux États-Unis, les think tanks fonctionnent en réalité sur un schéma de coopération public/privé : l'Urban Institute est né en 1968 de la volonté du gouvernement Johnson d'inclure dans sa politique la question urbaine; quant à la Brookings Institution, elle est aujourd'hui intimement liée à la politique de relance du Président Obama (Cynthia Ghorra-Gobin). Peut-être conviendraitil, dès lors, de considérer le monde des think tanks comme l'un de ces sous-espaces constitutifs du champ du pouvoir, dont l'imbrication aux sphères économiques et politiques s'exprimerait et se consoliderait à travers la circulation ininterrompue de certains agents sociaux. Les administrateurs multiples issus pour la plupart du secteur financier constituant en quelque sorte les effigies suprêmement individuelles d'un complexe français de think tanks relativement intégré (Geoffrey Geuens). 


\section{$\mathrm{N} \cdot \mathrm{O} \cdot \mathrm{T} \cdot \mathrm{E} \cdot \mathrm{S}$}

1. Le terme « think tank » est apparu à la charnière des années 1940-1950 pour qualifier le Rand Project, un centre de recherches en matière de défense né dans le giron de l'industrie aéronautique américaine.

2. Comme le rappelle fort justement Lucile Desmoulins, les boîtes à idées françaises spécialisées dans les relations internationales et les questions de sécurité ont «bénéficié du développement de procédures d'expertise pour le compte de la Délégation aux affaires stratégiques (DAS), inaugurée en 1992 au sein du ministère de la Défense, et du Centre d'analyse et de prévision (CAP), créé en 1973 au sein du ministère des Affaires étrangères » (voir article de Lucile Desmoulins dans le présent dossier).

3. Lucien Sfez, Critique de la communication, Paris, Seuil, 1988, p. 16.

4. Diane Stone, Andrew Denham et Mark Garnett (dir.), Think Tanks across Nations : A Comparative Approach, Manchester, Manchester University Press, 1998 ; James McGann et R. Kent Weaver (dir.), Think Tanks and Civil Societies, Piscataway, Transaction Publishers, 2000 ; Donald E. Abelson, Do Think Tanks Matter? Assessing the Impact of Public Policy Institutes, Kingston-Montréal, McGill-Queen's University Press, 2002 ; Diane Stone et Andrew Denham (dir.), Think Tank Traditions : Policy Research and the Politics of Ideas, Manchester, Manchester University Press, 2004. 
